\title{
Immunogenicity and antigenic relationships among spike proteins of porcine epidemic diarrhea virus subtypes G1 and G2
}

\author{
Xiaobo Wang ${ }^{1} \cdot$ Jianfei Chen $^{1} \cdot$ Da Shi $^{1} \cdot$ Hongyan Shi $^{1} \cdot$ Xin Zhang $^{1} \cdot$ \\ Jing Yuan ${ }^{1} \cdot$ Shibo Jiang $^{2} \cdot$ Li Feng $^{1}$
}

Received: 12 August 2015/Accepted: 16 November 2015/Published online: 26 November 2015

(C) Springer-Verlag Wien 2015

\begin{abstract}
Porcine epidemic diarrhea virus (PEDV) is a coronavirus that infects cells lining the small intestine of swine, resulting in vomiting, diarrhea, and dehydration. The amino acid sequence of the spike (S) protein, which is the principal target recognized by host immune cells, has multiple mutations that distinguish the two PEDV genotypes, G1 and G2. To determine whether these mutations lead to changes in antigenicity, as suggested by the failure of PEDV vaccines in China, we first optimized the codons of typical S genes of the CV777 vaccine strain (G1 subtype) and LNCT2 strain (G2 subtype) and expressed the recombinant full-length sequence of the $\mathrm{S}$ protein in a eukaryotic expression system. The IgG antibody levels of serum from mice immunized with purified $\mathrm{S}$ protein were markedly high. Antigenicity was compared by detection of polyclonal antibodies (PAbs) against the virus and S protein using an enzyme-linked immunosorbent assay (ELISA), an indirect immunofluorescence assay (IFA), and a serum cross-neutralization (SN) assay. Reactivity with the PAbs revealed significant cross-reactivity between the two PEDV subtypes, although there was a twofold difference in the antigenic responses based on PAb titers in the
\end{abstract}

X. Wang and J. Chen contributed equally to this work.

\section{Li Feng}

fl@hvri.ac.cn

1 Division of Swine Infectious Diseases, State Key Laboratory of Veterinary Biotechnology, Harbin Veterinary Research Institute, Chinese Academy of Agricultural Sciences, Harbin 150001, People's Republic of China

2 Key Laboratory of Medical Molecular Virology of Ministries of Education and Health, Shanghai Public Health Clinical Center and Shanghai Medical College, Fudan University, Shanghai 200032, People's Republic of China
ELISA and IFA. Consistent with the variation in the $\mathrm{S}$ gene sequences, the SN titer suggested differences in the neutralization activity of the $\mathrm{S}$ protein between the two subtypes, which could explain the antigenic variation between the PEDV subtypes G1 and G2.

\section{Introduction}

Porcine epidemic diarrhea virus (PEDV) is an acute and highly contagious enteric infectious disease characterized by vomiting, diarrhea, and dehydration in pigs of all ages, but especially in newborn piglets $[1,2]$. PEDV was first reported in England in 1971 [3] and then detected in Japan in 1982 and subsequently confirmed in other southeastern Asian countries [4]. In the USA, PEDV was first reported in 2013 and has since rapidly spread throughout the country [5, 6]. In China, the incidence of PEDV outbreaks has rapidly increased since 2010 , especially among newborn piglets aged from a few hours to one week, often resulting in death due to watery diarrhea and dehydration $[7,8]$. Although the use of inactivated and attenuated vaccines may have helped to reduce the prevalence of disease, PEDV has continually emerged, causing tremendous losses to the swine industry in China [9].

PEDV, a member of the genus Alphacoronavirus in the family Coronaviridae, has a single-stranded positive-sense RNA genome of approximately $28 \mathrm{~kb}$ encoding four structural proteins: the spike $(\mathrm{S})$, nucleocapsid $(\mathrm{N})$, envelope $(\mathrm{E})$, and membrane $(\mathrm{M})$ proteins $[10,11]$. The $\mathrm{S}$ protein is a glycoprotein consisting of 1383-1387 amino acids (aa) on the viral surface, which is composed of four regions, a signal peptide (aa 1-24), an extracellular region, a transmembrane domain (aa 1,334-1,356), and a short 
cytoplasmic tail (aa 1,357-1,383) [12]. Unlike other coronaviruses (i.e., murine coronavirus and bovine coronavirus), the $S$ protein of PEDV cannot be cleaved into $\mathrm{S} 1$ and S2 domains after PEDV maturation. Thus, the S1 domain (residues 1-789) and S2 domain (aa 790-1383) of PEDV are artificially defined based on homology to other coronavirus $S$ proteins $[13,14]$. The $S$ protein is responsible for binding and fusion of the virus to the host cells. It therefore not only determines cellular tropism but also plays a vital role in inducing production of neutralizing antibodies in the host $[15,16]$.

The $\mathrm{S}$ gene of PEDV is often used to evaluate the genetic diversity of coronaviruses [17, 18]. Based on phylogenetic analysis of the full-length $\mathrm{S}$ sequences isolated in China, PEDVs can be divided into two genotypes, G1 and G2 [19]. Furthermore, PEDV detected in China from 2010 to 2012 were mostly variant strains (most belonging to subtype G2) that differed genetically from the CV777 vaccine strain (belonging to subtype G1) [20]. However, many pig herds vaccinated with inactivated or attenuated CV777 vaccines still experienced high mortality rates among newborn piglets [17, 21]. Therefore, further evaluation of the immunogenicity and antigenic relationships of PEDV subtypes G1 and G2 is of vital importance. In the present study, we expressed the full-length $S$ gene in a eukaryotic expression system, prepared anti-S polyclonal antibodies (PAbs), and performed subsequent experiments to investigate differences in antigenicity between the PEDV subtypes to determine if mutations in the $S$ gene are the cause of immunization failure. The findings of this study also lay a foundation for further study of the function of the $S$ protein and to facilitate development of PAbs against PEDV.

\section{Materials and methods}

\section{Virus strains, cells, plasmids, and main reagents}

The PEDV G1 CV777 vaccine strain (GenBank accession number: KT323979) was preserved at Harbin Veterinary Research Institute (Harbin, China). PEDV G2 strain LNCT2 (GenBank accession number: KT323980) was isolated in Vero E6 cells in our laboratory. Vero E6 cells and HEK 293T cell were cultured in GIBCO ${ }^{\circledR}$ Dulbecco's modified Eagle medium (DMEM; Thermo Fisher Scientific, Inc., Waltham, MA, USA) with $10 \%$ fetal bovine serum (Thermo Fisher Scientific, Inc.) and maintained at $37{ }^{\circ} \mathrm{C}$ in a humidified atmosphere of $5 \% \mathrm{CO}_{2}$. The expression vector $\mathrm{pAAV}$-IRES-hrGFP was kindly provided by Professor Shibo Jiang (Fudan University, Shanghai, China). X-tremeGENE HP DNA Transfection Reagent was purchased from Roche Diagnostics GmbH (Mannheim,
Germany). Mouse monoclonal Anti-Flag ${ }^{\circledR}$ antibody produced in mouse and fluorescein isothiocyanate (FITC)conjugated goat anti-mouse IgG were purchased from Sigma-Aldrich Corporation (St. Louis, MO, USA). AntiDYKDDDDK G1 Affinity Resin was purchased from GenScript USA, Inc. (Piscataway Township, NJ, USA). A BCA Protein Assay Kit was purchased from Thermo Fisher Scientific, Inc. Horseradish peroxidase (HRP)-conjugated goat anti-mouse IgG was purchased from ZSGBBIO (Beijing, China), and IRDye@680RD goat anti-mouse IgG was purchased from LI-COR Biosciences (Lincoln, NE, USA).

\section{$S$ gene sequences and phylogenetic analysis}

Full-length S sequences of the PEDV CV777 vaccine strain, the classical Chinese strain $\mathrm{CH} / \mathrm{S}$, and 20 epidemic Chinese strains isolated from 2011 to 2015 were used for sequence alignments and phylogenetic analysis. Homology among multiple aa sequences was analyzed using MegAlign sequence alignment software (DNASTAR, Inc., Madison, WI, USA). A phylogenetic tree was constructed from the aligned nucleotide sequences by the maximumlikelihood method using MEGA 5.05 statistical analysis software (http://www.megasoftware.net/).

\section{Construction of expression vectors}

To improve the expression levels of the $\mathrm{S}$ protein, $\mathrm{S}$ gene sequences were codon optimized with the strategy of 'one amino acid-one codon', in which the most preferred codon of the host for a given amino acid is used in the target sequence without changing the amino acid sequences [22]. The nucleotide sequences of the CV777 and LNCT2 S genes were edited in accordance with the codon table for Homo sapiens in the Codon Usage Database (http://www. kazusa.or.jp/codon/) for optimal expression in HEK 293T cells and biochemically synthesized for CV777-S and LNCT2-S by Beijing Genomics Institute (Beijing, China). Full-length sequences of the $\mathrm{S}$ gene were cloned between the BamHI and XhoI restriction sites in the pAAV-IREShrGFP plasmid. The recombinant plasmids pAAV-opti CV777 S-flag and pAAV-opti-LNCT2 S-flag were extracted using a Plasmid Maxi Kit (QIAGEN, Hilden, Germany).

\section{Expression of recombinant full-length $S$ protein}

HEK 293T cells were cultured to $60 \%$ confluency in a sixwell tissue culture plate at $37{ }^{\circ} \mathrm{C}$ and then transfected with $2 \mu \mathrm{g}$ of pAAV-opti CV777 S-flag and pAAV-opti LNCT2 S-flag together with $2 \mu \mathrm{L}$ of transfection reagent. After 48 h, the HEK 293T cells were collected, treated with IP 
Lysis Buffer (Thermo Fisher Scientific, Inc.), and centrifuged at $12,000 \mathrm{rpm}$ for $30 \mathrm{~min}$. Next, proteins in the supernatants were separated by sodium dodecyl sulfate polyacrylamide gel electrophoresis (SDS-PAGE) on $10 \%$ gels and transferred to a polyvinylidene difluoride (PVDF) membrane for $60 \mathrm{~min}$. The PVDF membrane was blocked with $5 \%$ skim milk for $2 \mathrm{~h}$ at $37{ }^{\circ} \mathrm{C}$, incubated with mouse anti-flag antibody (dilution, $1: 5,000$ ) at $37{ }^{\circ} \mathrm{C}$ for $1 \mathrm{~h}$, and then further incubated with IRDye 680RD goat anti-mouse IgG (dilution, $1: 4,000$ ) at $37{ }^{\circ} \mathrm{C}$ for $45 \mathrm{~min}$. The protein bands were visualized using an Odyssey ${ }^{\circledR}$ CLx Infrared Imaging System (LI-COR Biosciences).

\section{Recombinant $\mathbf{S}$ protein purification}

HEK 293T cells were cultured in $75-\mathrm{cm}^{2}$ bottles and transfected with $20 \mu \mathrm{g}$ of pAAV-opti PEDV-S-flag plasmids. At $48 \mathrm{~h}$ post-transfection, the cell lysate was harvested as described above, recombinant $\mathrm{S}$ protein was purified using Anti-DYKDDDDK G1 Affinity Resin, the cell lysate was loaded onto a column, and the soluble proteins were immunoprecipitated with Anti-Flag Resin. The purified proteins were concentrated using Amicon Ultra centrifugal filters (molecular weight cutoff, $100 \mathrm{~K}$; EMD Millipore, Billerica, MA, USA). Protein concentrations were measured using the bicinchoninic acid (BCA) protein assay, and the final products were analyzed by SDS-PAGE to confirm purification of the target protein.

\section{Preparation of anti-S protein PAbs}

All animal procedures were approved by the Ethics Committee of Harbin Veterinary Research Institute. Three groups of 6-week-old female pathogen-free BALB/c mice were respectively immunized subcutaneously three times, at two-week intervals, with $50 \mu \mathrm{g} /$ mouse of recombinant CV777 S protein, LNCT2 S protein formulated in Freund's adjuvant (Sigma-Aldrich Corporation) and phosphate-buffered saline (PBS) which was used as a negative control. Blood was collected from the tail vein of three mice from each group, and serum samples were prepared prior to the first immunization and at one-week intervals, then stored frozen until assayed.

\section{Detection of IgG antibodies of immunized mice using an enzyme-linked immunosorbent assay (ELISA)}

IgG antibodies of the immunized mouse's serum samples were detected by ELISA [23]. Ninety-six-well plates Costar $^{\mathrm{TM}}$; Thermo Fisher Scientific, Inc.) were coated with the purified recombinant CV777 and LNCT2 S proteins (200 ng/well) in coating buffer $\left(20 \mathrm{mM} \mathrm{Na}_{2} \mathrm{CO}_{3}\right.$,

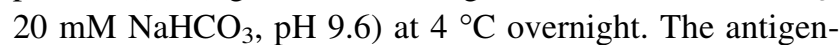
coated plates were washed three times with PBS with Tween ${ }^{\circledR} 20$ (PBST) and blocked with $200 \mu \mathrm{L}$ of $5 \%$ skim milk for $1 \mathrm{~h}$ at $37{ }^{\circ} \mathrm{C}$. The blocked wells were washed three times with PBST. Anti-S $\mathrm{S}_{\mathrm{LNCT2}}$ or anti-S $\mathrm{S}_{\mathrm{CV} 777}$ polyclonal antisera (PAbs) collected at different time points were diluted from 1:200 to 1:204,800 with PBS and added to the wells. The plates were incubated at $37{ }^{\circ} \mathrm{C}$ for $1 \mathrm{~h}$, washed three times with PBST, and HRP-conjugated goat antimice $\operatorname{IgG}(1: 5000)$ was added to each well. The plates were incubated at $37{ }^{\circ} \mathrm{C}$ for $45 \mathrm{~min}$ and washed three times with PBST. Then, $100 \mu \mathrm{L}$ of ABTS [2,2'-azinobis-(3-ethylbenzothiazoline-6-sulfonic acid)] peroxidase substrate was added to each well, and the plates were incubated for an additional $20 \mathrm{~min}$ at $37^{\circ} \mathrm{C}$ in the dark. The reaction was stopped by the addition of $1 \mathrm{M} \mathrm{NaF}$, and the absorbance was read at a wavelength of $405 \mathrm{~nm}\left(\mathrm{OD}_{405}\right)$ using an enzyme-linked immunosorbent assay (ELISA) plate reader.

\section{Indirect immunofluorescence assay (IFA)}

Confluent monolayers of Vero E6 cells in 24-well plates were washed three times with PBS. Subsequently, each well was incubated with $10^{4}$ times the median tissue culture infective dose $\left(\mathrm{TCID}_{50}\right)$ of strain CV777 and/or LNCT2 in DMEM supplemented with $10 \mu \mathrm{g}$ of trypsin per ml. After incubation at $37{ }^{\circ} \mathrm{C}$ for $48 \mathrm{~h}$, monolayers were rinsed with PBS and fixed with $4 \%$ paraformaldehyde at $4{ }^{\circ} \mathrm{C}$ for $30 \mathrm{~min}$. They were then washed twice with PBS and incubated with $0.2 \%$ TritonX-100 at $37{ }^{\circ} \mathrm{C}$ for $20 \mathrm{~min}$. After washing with PBS, the wells were blocked with $1 \%$ bovine serum albumin at $37{ }^{\circ} \mathrm{C}$ for $2 \mathrm{~h}$, washed three times with PBS, and incubated with $\mathrm{CV} 777$ or LNCT2 anti-S PAbs at $37{ }^{\circ} \mathrm{C}$ for $1 \mathrm{~h}$. To determine titers, serum samples were initially diluted 400 -fold and then serially diluted twofold and rinsed three times with PBS. Fluorescein-isothiocyanate-conjugated goat anti-mouse IgG at a dilution of 1:2,000 in PBS was added to each well. After 45 min of incubation in the dark at $37^{\circ} \mathrm{C}$, the plates were washed three times with PBS and incubated with $4^{\prime}, 6-$ diamidino-2-phenylindole at a dilution of 1:1,000 for $15 \mathrm{~min}$ in the dark at $37{ }^{\circ} \mathrm{C}$. After washing three times with PBS, the samples were examined using a digital inverted microscope (Thermo Fisher Scientific, Inc.).

\section{Western blot analysis}

The cross-reactivity between two types of virus $\mathrm{S}$ protein and anti-S protein PAbs were assessed by western blot analysis. Briefly, Vero E6 cells in $75-\mathrm{cm}^{2}$ bottles were infected with either the CV777 or LNCT2 virus 
Fig. 1 Phylogenetic analysis based on the $\mathrm{S}$ sequences of 28 porcine epidemic diarrhea virus (PEDV) strains. A maximumlikelihood tree was constructed using MEGA 5.05 software. The GenBank accession numbers of PEDV reference strains are shown in brackets. The LNCT2 and CV777 vaccine strains used in this study are enclosed in a red box (color figure online)

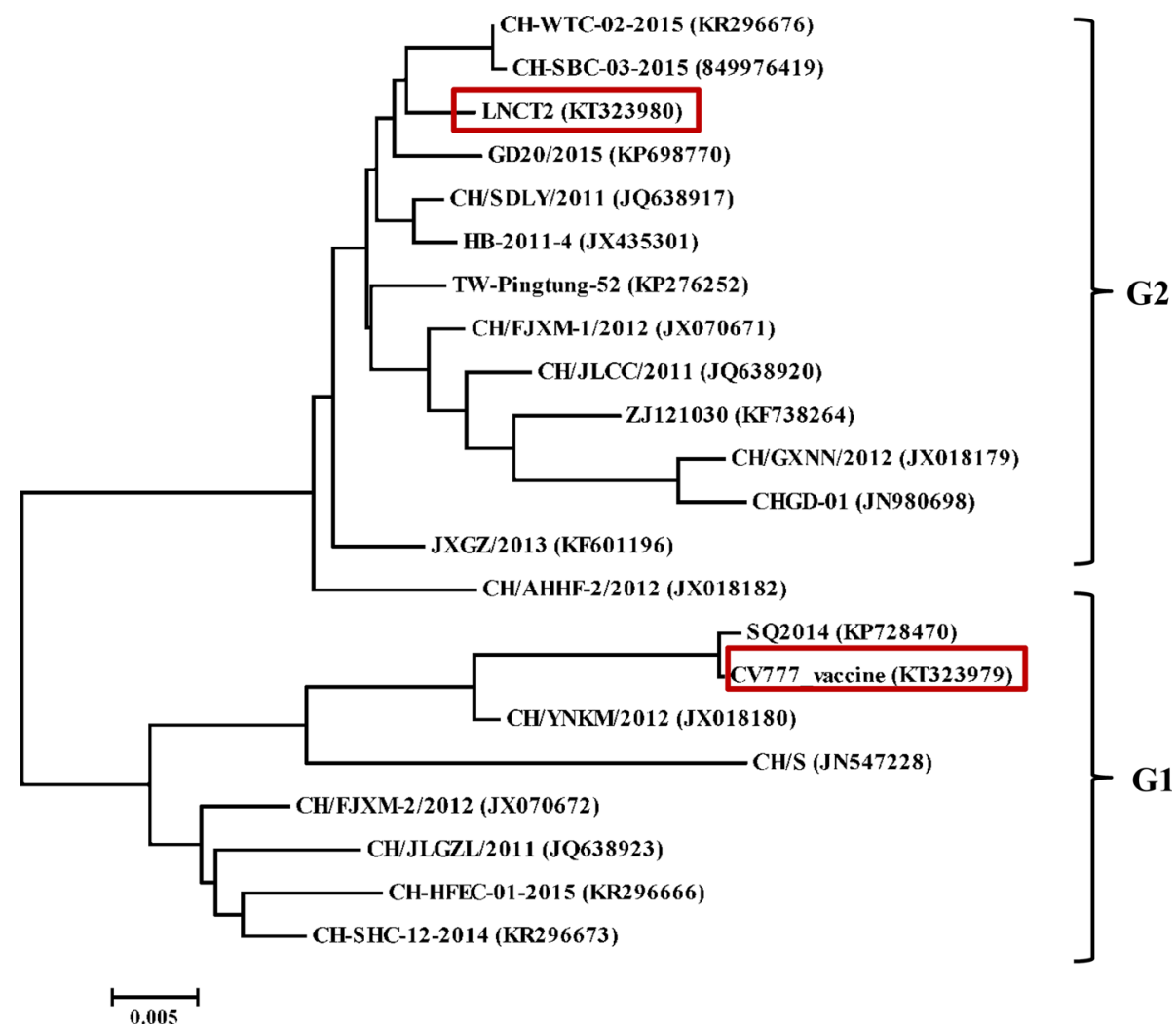

(multiplicity of infection $=1$ ) for $48 \mathrm{~h}$, treated with Mammalian Protein Extraction Reagent (Thermo Fisher Scientific, Inc.) for $20 \mathrm{~min}$, and centrifuged at 12,000 $\mathrm{g}$ for $30 \mathrm{~min}$. The supernatants containing total virus proteins were subjected to $10 \%$ SDS-PAGE followed by western blot analysis as described above with anti-S PAbs diluted to $1: 1,000$.

\section{Cross serum neutralization (SN) test}

The SN test was performed according to the fixed-virusdilution serum method described by Reed and Muench [24]. Briefly, confluent monolayers of Vero E6 cells in 96-well plates were washed three times with DMEM. PAbs against the $\mathrm{S}$ protein were inactivated at $56{ }^{\circ} \mathrm{C}$ for $30 \mathrm{~min}$ and then diluted twofold starting at 1:25. They were then mixed with the same volume $(50 \mu \mathrm{L})$ of $200 \mathrm{TCID}_{50}$ of virus diluted with DMEM supplemented with $10 \mu \mathrm{g}$ of trypsin per $\mathrm{ml}$ and incubated at $37{ }^{\circ} \mathrm{C}$ for $1 \mathrm{~h}$. Subsequently, $0.1 \mathrm{ml}$ of each virus-serum mixture was inoculated onto Vero E6 cell monolayers in 96-well tissue culture plates. After 5 days, specific cytopathic effects (CPEs) of cells were observed under an inverted microscope. SN titers were expressed as the titer of the highest serum dilution resulting in $50 \%$ inhibition of PEDV infection.

\section{Statistical analysis}

One-way analysis of variance was used to determine statistical differences between groups. All statistical analysis was performed using GraphPad Prism version 5.0 software (GraphPad Software, Inc., La Jolla, CA, USA). Probability ( $p)$ values of $<0.05$ and $<0.01$ were considered statistically significant and highly statistically significant, respectively.

\section{Results}

\section{Phylogenetic analysis based on $\mathrm{S}$ sequences}

The maximum-likelihood phylogenetic relationships inferred from the aa sequences of the $S$ protein confirmed that the two genotypes were PEDV strains G1 and G2. The CV777 vaccine strain was subtype G1, while the LNCT2 strain was subtype G2 (Fig. 1). Comparisons of the aa sequences of the $\mathrm{S}$ protein showed that the G1 subtypes shared $90.2 \%-95.8 \%$ sequence identity with the G2 subtypes. The S sequence of strain LNCT2 shared $96.3 \%$ $98.8 \%$ sequence identity with other G2 subtypes, indicating that LNCT2 is a typical PEDV G2 strain. The CV777 vaccine strain shared $92.3 \%$ sequence identity with LNCT2 strains with multiple aa mutations (Table 1). 
Table 1 Amino acids sequence comparisons of spike protein of G1 and G2 subtype strains

\begin{tabular}{llllll}
\hline Percentage identity & PEDV strain & \multicolumn{5}{l}{ Percentage identity } \\
\cline { 3 - 6 } & & G1 & G2 & CV777 vaccine & LNCT2 \\
\hline G1 & $95.4-99.5$ & $90.2-95.8$ & $95.4-99.5$ & $92.3-95.4$ \\
G2 & $90.2-95.8$ & $95.7-99.9$ & $90.4-93.8$ & $96.3-98.8$ \\
CV777 vaccine & $95.4-99.5$ & $90.4-93.8$ & 100 & 92.3 \\
LNCT2 & $92.3-95.4$ & $96.3-98.8$ & 92.3 & 100 \\
\hline
\end{tabular}

Sequence homology between different subtypes was analyzed by DNASTAR software using the MegAlign program

\section{Expression and purification of full-length recombinant PEDV S proteins}

HEK 293T cells in six-well tissue culture plates were transfected with recombinant plasmids (pAAV-CV777 S-flag and pAAV-LNCT2 S-flag) containing unoptimized $\mathrm{S}$ genes and recombinant plasmids (pAAV-opti CV777 S-flag and pAAV-opti-LNCT2 S-flag) containing optimized $\mathrm{S}$ genes. As the $\mathrm{S}$ protein was co-expressed with the hrGEP protein, the fluorescence intensity reflected the expression level of the S protein. We found that the expression levels of the $S$ proteins were significantly increased by codon optimization (Fig. 2A). S proteins were detected by western blotting using anti-Flag mouse monoclonal antibody (MAb) as the primary antibody. The results showed that the recombinant proteins reacted specifically with the primary antibody, and each had a molecular weight of $250 \mathrm{kDa}$ (Fig. 2B). These results were in accordance with those of the SDS-PAGE analysis with the purified $\mathrm{S}$ protein (Fig. 2C).

\section{Detection of IgG antibodies in serum of mice immunized with $S$ protein}

Specific IgG antibodies were detected in serum samples collected at different time points using an indirect ELISA, as described previously. A shown in Fig. 3, IgG antibody levels against the $\mathrm{S}$ protein in serum from immunized mice were significantly increased at 14 days post-immunization $(p<0.05)$ and then highly significantly increased at 21 days, as compared with mice immunized with PBS $(p<0.01)$, while there were no significant differences in titers of IgG antibodies induced by CV777 and LNCT2 S proteins at different time points $(p>0.05)$.

\section{Assessment of antigenic cross-reaction between G1 and G2 $S$ proteins using an ELISA}

The ELISA results indicated high cross-reactivity between the PAbs and the two types of PEDV S protein. The titers of anti-S PAbs reacting with same subtype $\mathrm{S}$ protein reached 1:204,800, while the titers reacting with different subtype $S$ proteins reached 1:102,400. As shown in Fig. 4A and $\mathrm{B}$, the $\mathrm{OD}_{405}$ values of anti-S $\mathrm{S}_{\mathrm{CV} 777}$ protein $\mathrm{PAbs}$ at different dilutions reacting with the CV777 S protein were much higher than those reacting with the LNCT2 S protein.

\section{Assessment of antigenic cross-reaction between G1 and G2 strains using an IFA}

The IFA results indicated that PEDV $S$ protein was present in the cytoplasm of Vero E6 cells (Fig. 5A). Both PEDV CV777 and LNCT2 could induce obvious CPE: Vero E6 cell fused with each other and formed syncytia. The structure of cells infected by LNCT2 was still clear, while CV777-infected cells were all fused and no single cellular morphology was generally observed. The syncytia of CV777-infected cells were larger than those of LNCT2infected cells (Fig. 5A and B).

The IFA results confirmed that the anti-S PAbs crossreacted with PEDV strains CV777 and LNCT2. Next, we detected the fluorescence intensity of the antigenic crossreaction between the the anti-S protein PAbs and the two PEDV subtypes. Consistent with the ELISA results, which showed twofold differences between different PEDV subtypes based on titers of the anti-S PAbs, the highest titer of anti-S PAbs reacting with PEDV within subtypes (observing green fluorescence) reached 1:51,200, while the reaction of anti-S PAbs and PEDV across subtypes only reached 1:25,600 (Table 2).

\section{Assessment of antigenic cross-reaction between G1 and G2 strains using a western blot assay}

Anti-S PAbs bound with the PEDV S protein, which was about $220 \mathrm{kDa}$, slightly smaller than the recombinant $\mathrm{S}$ protein, as was expected. The results of the western blot assay demonstrated that the anti-S PAbs cross-reacted with strains CV777 and LNCT2 (Fig. 6A and B), indicating that PEDV $S$ proteins had at least one common antigenic epitope across the G1 and G2 strains. 
Fig. 2 Expression and purification of recombinant $S$ protein. (A) $\mathrm{S}$ protein expression levels were increased by codon optimization. (1) HEK293T cells transfected with the pAAV-CV777 S-flag plasmid. (2) HEK 293T cells transfected with the pAAV-opti CV777 S-flag plasmid. (3) HEK293T cells transfected with the pAAV-LNCT2 S-flag plasmid. (4) HEK 293T cells transfected with the pAAV-opti LNCT2 S-flag plasmid. The cells were examined using a fluorescence microscope at $100 \times$ magnification. (B) Western blot analysis of recombinant $\mathrm{S}$ proteins. (1) Protein marker. (2) Recombinant CV777 S protein.

(3) Recombinant LNCT2 S protein. (4) Negative control. (C) Purification of recombinant $S$ proteins. (1) Protein marker. (2) Total cells lysate of HEK 293T cells transfected with the paav-opti CV777 S-flag plasmid. (3) Purified CV777 S protein using Anti-Flag Affinity Resin. (4) Total cell lysate of HEK 293T cells transfected with the paav-opti LNCT2 S-flag plasmid. (5) Purified LNCT S protein using Anti-Flag Affinity Resin
A

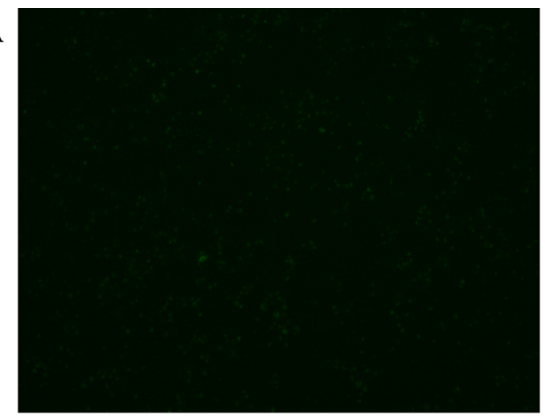

1

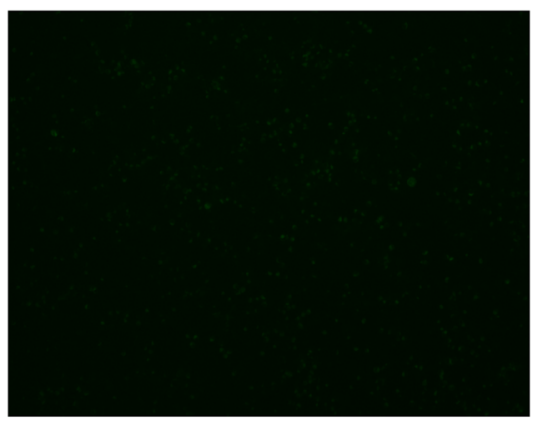

3

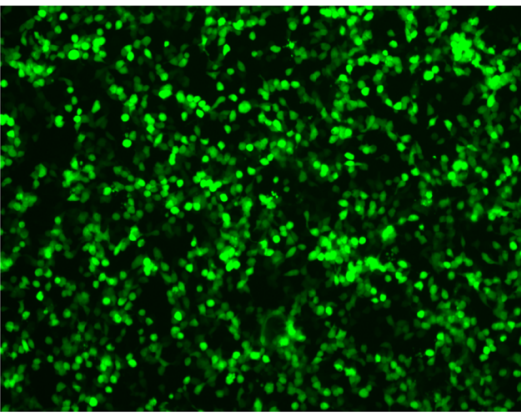

2

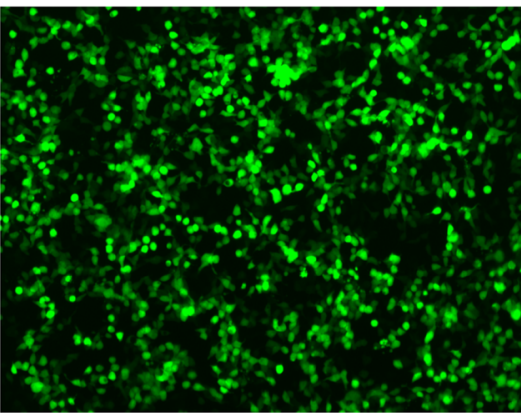

4

B

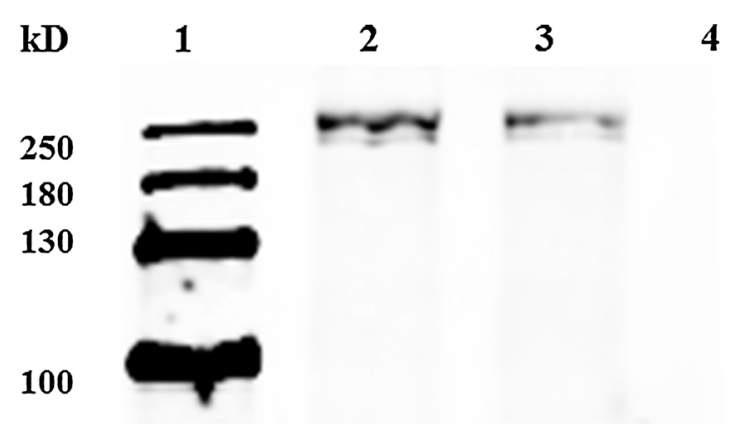

C

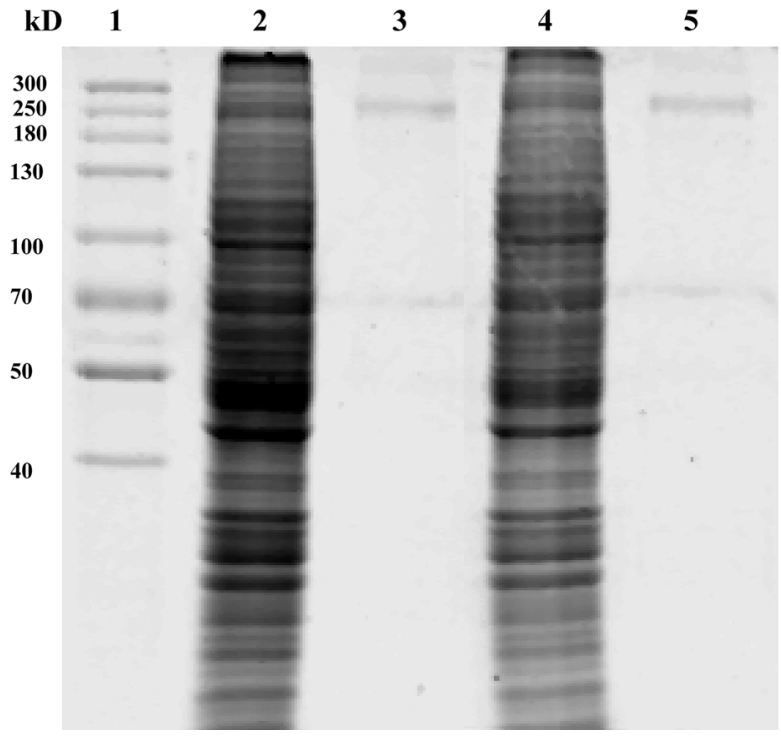




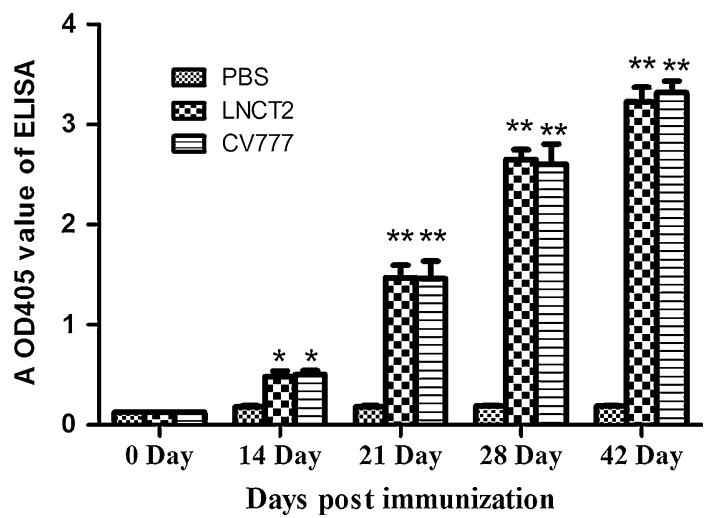

Fig. 3 Serum $\operatorname{IgG}$ antibodies of mice immunized with the $S$ protein. All antisera were diluted to 1:400 with PBS. The y-axis represents the IgG antibody titer index $\left(\mathrm{OD}_{405}\right)$. *, significant difference as compared to the PBS-immunized group $(p<0.05)$. **, highly significant difference as compared to the PBS immunized group $(p<0.01)$

\section{SN assay}

The titers of anti-S PAbs neutralizing PEDV were calculated by the Kärber method. The average titer of anti$\mathrm{S}_{\mathrm{CV} 777} \mathrm{PAb}$, which protected $50 \%$ of CV777-infected cells, was 1:336, while the titer cross-neutralizing LNCT2 was $1: 150$. The average titer of anti- $S_{\mathrm{LNCT} 2}$ PAbs neutralizing LNCT2 reached 1:230, while the titer of neutralizing CV777 was 1:387 (Fig. 7). The SN titer of anti$\mathrm{S}_{\mathrm{CV} 777}$ PAbs neutralizing CV777 strain was more than twofold higher than those neutralizing the LNCT2 strain, while the neutralizing titers of anti- $\mathrm{S}_{\mathrm{LNCT} 2} \mathrm{PAbs}$ were less than twofold of those reacting with the two strains.

\section{Discussion}

The ongoing epidemic of PEDV has resulted in significant economic losses to the swine industry in Asia as well as Europe and North America. In China, mortality due to PEDV infection can reach $80 \%-100 \%$ in piglets less than 10 days old [25]. In the USA, PEDV infection has resulted in a loss of almost $10 \%$ of the domestic pig population after only a 1-year epidemic period [26]. Located on the surface of PEDV, the $\mathrm{S}$ protein plays an pivotal role in recognizing receptors of target cells, thereby inducing production of neutralizing antibodies by activated host immune cells $[15,16]$. Although there have been relatively few studies using full-length sequences of the PEDV $S$ protein, most investigating the immunogenicity of the PEDV S protein have focused on the $S 1$ region [27-29]. As PEDV $S$ protein cannot be cleaved into $S 1$ and $S 2$ domains after virus maturation and S2 domain may also have potential neutralizing linear and conformational epitopes,
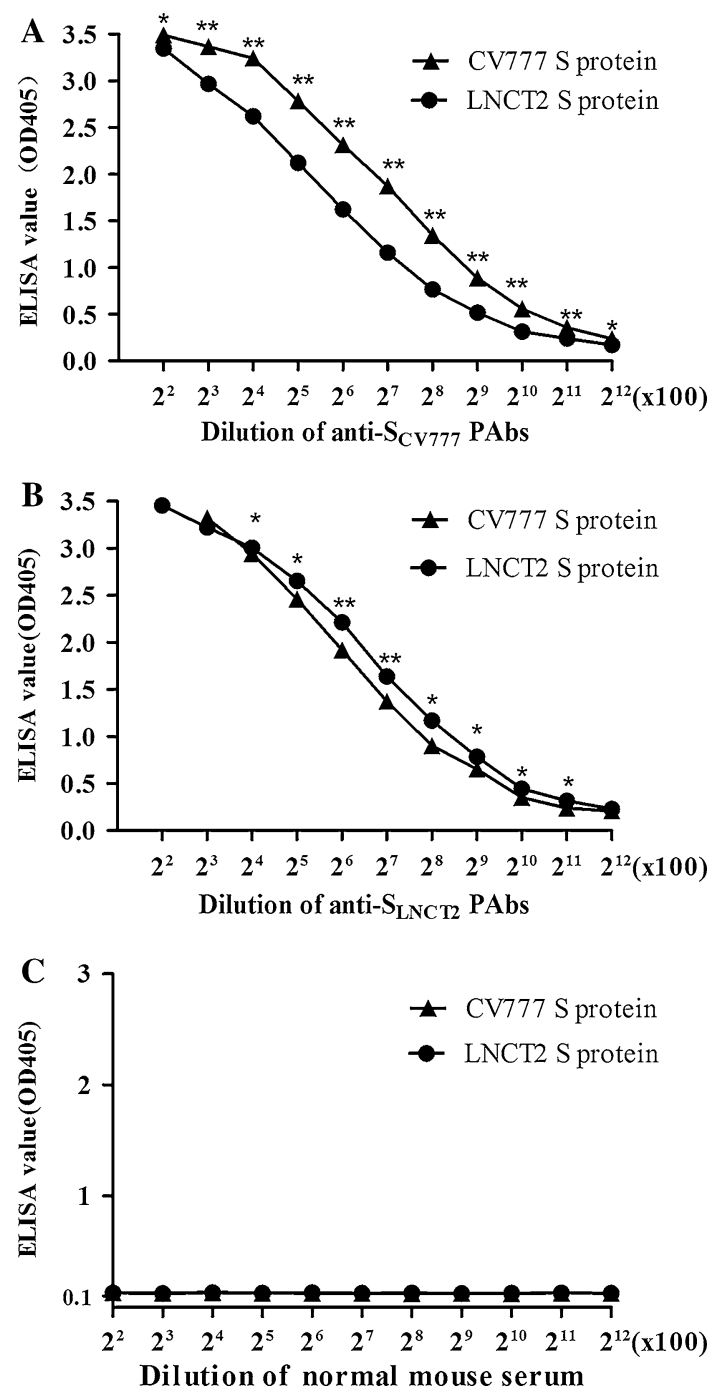

Fig. 4 ELISA results of the reaction between anti-S PAbs and the S proteins of two PEDV subtypes. (A) Dilution of anti-S $\mathrm{CV}_{\mathrm{C} 777} \mathrm{PAbs}$ reacting with the $S$ proteins of two PEDV subtypes. (B) Dilution of anti- $S_{\text {LNCT2 }}$ PAbs reacting with the $S$ proteins of the two PEDV subtypes. (C) Dilution of mouse serum before immunization reacting with the $\mathrm{S}$ proteins of the two PEDV subtypes

the whole $\mathrm{S}$ protein with its native conformation might be a better immunogen than the S1 protein. Previously, severe acute respiratory syndrome coronavirus (SARS-CoV) and Middle East respiratory syndrome coronavirus (MERS$\mathrm{CoV}$ ) spike proteins have been shown to produce high-titer antibodies in mice [30]. In the present study, we successfully expressed and purified the full-length $\mathrm{S}$ protein using a eukaryotic expression system. Western blot analysis confirmed that the $\mathrm{S}$ protein was specifically detected by anti-Flag antibodies (Fig. 1B). The IFA indicated that the $\mathrm{S}$ protein was expressed at higher levels in HEK 293T cells through codon optimization (Fig. 1A), as demonstrated with other coronaviruses [31]. 
Fig. 5 IFA of the anti-S PAbs in Vero E6 cells against the $S$ proteins of the two PEDV subtypes. (A) Anti-S $\mathrm{CV}_{\mathrm{C} 77} \mathrm{PAbs}$ (1:800 dilution) reacted against CV777-infected Vero E6 cells. (B) Anti-S $\mathrm{CV}_{\mathrm{C} 77}$ PAbs (dilution, 1:800) against LNCT2-infected Vero E6 cells. The cells were examined using a fluorescence microscope at $200 \times$ magnification
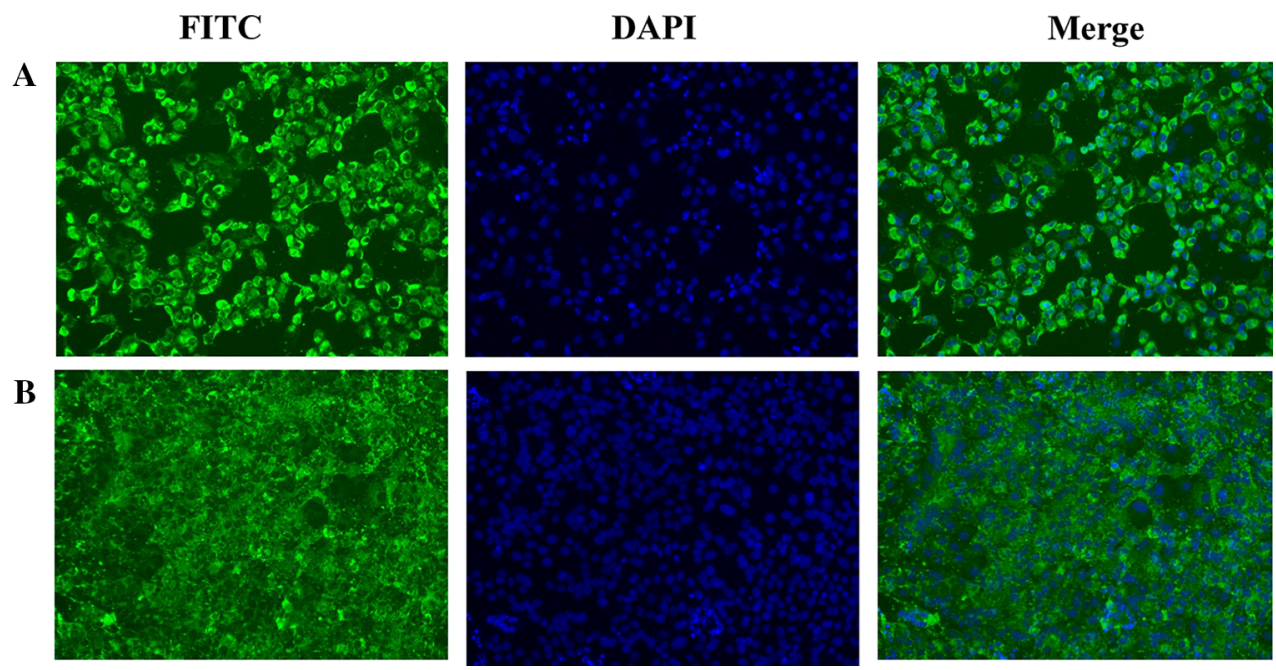

Table 2 Luminescence intensity of fluorescence of anti-S polyclonal antibodies against different types of virus

\begin{tabular}{lllllllll}
\hline & \multicolumn{7}{c}{ Dilution of anti-CV777 S polyclonal antiserum } \\
\cline { 2 - 8 } & 1600 & 3200 & 6400 & 12800 & 25600 & 51200 & 102400 \\
\hline CV777 & +++ & +++ & ++ & ++ & + & + & - \\
LNCT2 & +++ & +++ & ++ & ++ & + & - & - \\
Vero E6 & - & - & - & - & - & - & - \\
\hline \multicolumn{7}{c}{ Dilution of anti-LNCT2 S polyclonal antiserum } \\
\cline { 2 - 8 } \\
\cline { 2 - 8 } & 1600 & 3200 & 6400 & 12800 & 25600 & 51200 & 102400 \\
\hline CV777 & +++ & +++ & ++ & ++ & + & + & - \\
LNCT2 & +++ & +++ & ++ & ++ & + & - & - \\
Vero E6 & - & - & - & - & - & - & - \\
\hline
\end{tabular}

The luminescence intensity was divided into four degrees: +++ , strong; ++, moderate; +, weak; -, negative

The PEDV S protein has 27-30 potential glycosylation sites that help to maintain its biological function [32]. Therefore, we chose a eukaryotic expression system that was particularly useful for the production of such a large multi-domain protein requiring complex folding machinery and post-translational modifications [33] so that the structure of the $\mathrm{S}$ protein was close to the naturally occurring conformation. Other advantages of choosing a eukaryotic expression system were that the expressed fusion protein was soluble as the biological function was maintained [34, 35]. In the present study, we established a method to express and purify the PEDV S protein, which can be used not only to evaluate immunogenicity but also to study $\mathrm{S}$ protein function. The IgG levels of mice immunized with the $\mathrm{S}$ protein were significantly increased beginning on post-immunization day 14 , and antibody titers reached
1:204,800 on post-immunization day 42 according to the ELISA results (Fig. 2). The high IgG antibody titers of immunized mice indicated that purified $\mathrm{S}$ protein may be a good candidate as an immunogen for PAb production and diagnostic methods.

Since 2010, PEDV outbreaks in several provinces have caused significant economic losses to the swine industry in China [7, 8]. Although a CV777-inactivated vaccine has been used to immunize swine herds against PEDV infection, its efficacy has been rather low [8]. Therefore, it is of vital importance to develop a CV777 vaccine that can cross-protect against epidemic strains of the G2 subtype. Previous studies have found differences in the antigenicity between PEDV CV777 and strains isolated in the USA, and some pig antibodies and MAbs showed 4- to 16-fold differences between the titers of the homologous and heterologous strains CCIF and VN, as at least one epitope on the N-terminal region of PEDV/TGEV N protein contributed to this cross-reactivity [36]. However, the MAbs used in the previous study were against the $\mathrm{N}$ protein, which is not the major antigen that induces production of neutralizing antibodies by host cells. In our previous study, we also found twofold differences in pig antibodies based on SN titers between strains CV7777 and LNCT2 (data not shown). A comparison of sequences of two types of PEDV G2 S protein from 14 recent Chinese epidemic strains from 2011 to 2015 showed that they had $90.4 \%-93.8 \%$ sequence identity to the CV777 vaccine strain (Table 1), and sequence variations were concentrated in the $\mathrm{N}$-terminal region of the $\mathrm{S}$ protein due to a point mutation, a deletion, and an insertion of four amino acids. The LNCT2 strain we chose belonged to the G2 subtype, and its S sequence was $92.3 \%$ identical to that of the CV777 vaccine strain (G1 subtype). The neutralizing epitope domain COE (aa 499-638) had eight different amino acids, and the 
Fig. 6 Western blot analysis of the antigenic relationships between the CV777 and LNCT2 strains. (A) Anti-S $\mathrm{CV}_{777}$ PAbs used as the primary antibody to detect the $S$ proteins of the two PEDV subtypes. (B) Anti$\mathrm{S}_{\mathrm{LNCT} 2}$ PAbs used as the primary antibody to detect the $S$ proteins of the two PEDV subtypes. (M) Protein marker. (1) Total protein of Vero E6 cells incubated with strain CV777. (2) The total protein of Vero E6 cells incubated with strain LNCT2. (3) The total protein of Vero E6 cells
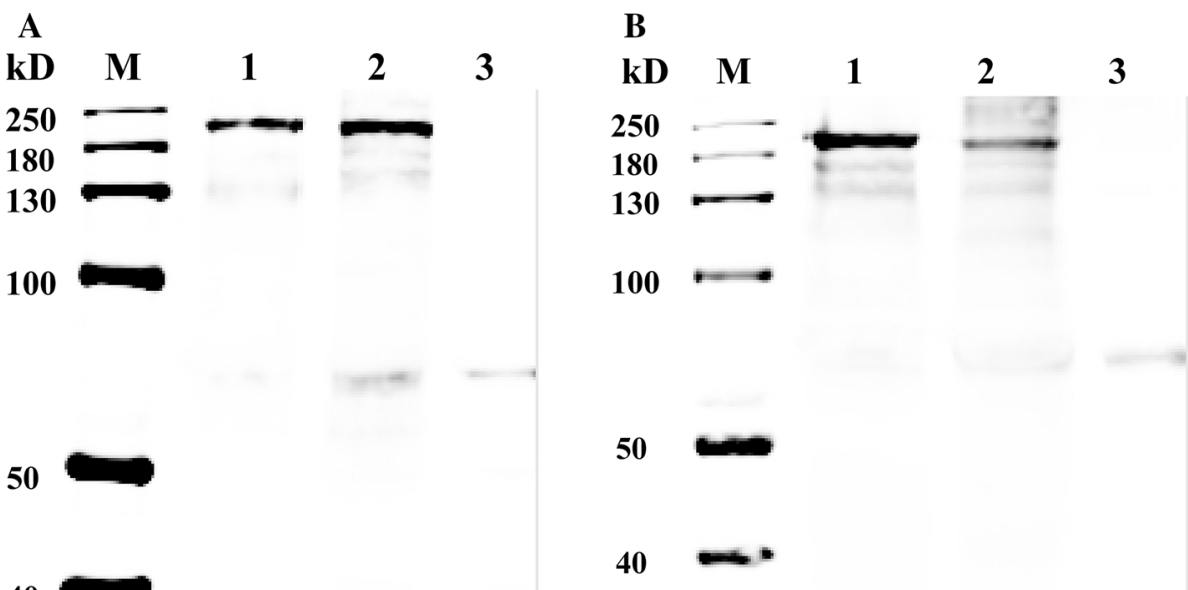

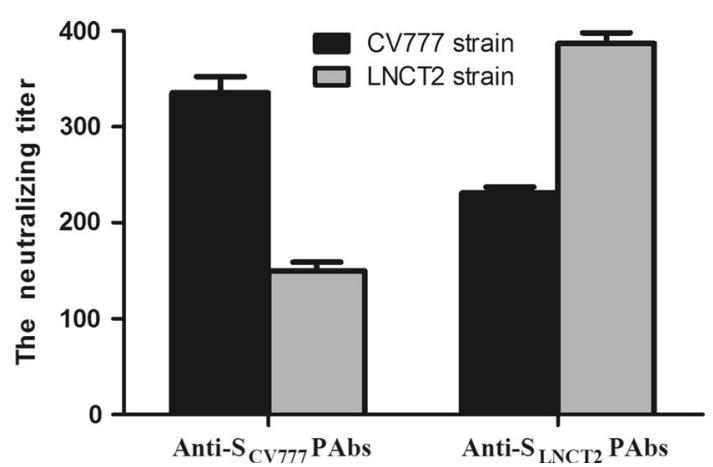

Fig. 7 Cross serum neutralization (SN) between the S proteins of the two PEDV subtypes and anti-S PAbs. Reciprocals of PEDV neutralizing antibody titers were expressed as the dilution inhibiting PEDV infection by $50 \%$, which was calculated as follows: $\log 50 \%$ neutralizing titer $=\mathrm{L}-\mathrm{d}(\mathrm{s}-0.5)$, where $L$ is the $\log$ of the lowest dilution factor, $d$ is the difference between the dilution factors, and $s$ is the sum of the ratios of positive wells

epitope motif SS6 (764LQDGQVKI771) had two aa mutations between the two PEDV subtypes [19]. Based on these differences in $\mathrm{S}$ protein sequences, we investigated whether mutations in the $\mathrm{S}$ protein resulted in a change in antigenicity between strains G1 and G2.

The ELISA and IFA results showed significant antigenic cross-reactivity between the two PEDV subtypes. However, there was approximately a twofold difference in the antigenic responses based on the titers of the PAbs. Especially, the ELISA results showed that reactivity of the anti$\mathrm{S}_{\mathrm{CV} 777}$ PAbs at different dilutions with the CV777 S protein was much greater than that with the LNCT2 S protein, indicating that the ability of anti-S $\mathrm{S}_{\mathrm{CV} 777}$ PAbs to crossreact with different subtypes was lower than that of the anti- $\mathrm{S}_{\mathrm{LNCT} 2}$ PAbs. In addition, previous studies have shown that the PEDV CV777 strain induces larger syncytia than Korean strain DR13 and three US strains [36-38]. Comparing the IFA results of the CV777 and LNCT strains, we also found a similar phenomenon (Fig. 7). These results indicated that CV777 and epidemic strains may have differences in their ability to enter cells and induce fusion and may have different effects on cell morphology.

The results of western blot analysis indicated that the PEDV S proteins had at least one common antigenic epitope, allowing the S protein PAbs to cross-react with different PEDV subtypes. Likewise, the results of the SN assay confirmed the presence of high titers of PAbs against the $\mathrm{S}$ protein that were able to cross-neutralize other PEDV subtypes. However, consistent with the ELISA and IFA results, there were obvious differences in serum neutralizing responses across subtypes. The SN titer of anti$\mathrm{S}_{\mathrm{CV} 777}$ PAbs neutralizing strain CV777 was more than twofold higher than the PAbs neutralizing strain LNCT, while the differences in the neutralizing titers of anti$\mathrm{S}_{\mathrm{LNCT2}}$ PAbs across subtypes was less than twofold. These results indicate that the neutralizing epitopes of the LNCT2 $S$ protein had changed or that new neutralizing epitopes had emerged due to mutations in the $\mathrm{S}$ protein. In the subsequent experiments, we found that the 5F7 monoclonal antibody against the LNCT S protein could react with strain LNCT, but not strain CV777 (data not shown). Further studies to identify the MAb 5F7 epitope are underway.

In summary, the full-length sequence of the PEDV $S$ gene was codon-optimized and successfully expressed in HEK293T cells. The findings of this study confirmed that the recombinant $\mathrm{S}$ protein was highly immunogenic in 
mice and could effectively induce production of antibodies, especially neutralizing antibodies. Furthermore, we found significant antigenic relationships between the PEDV $S$ protein of subtypes G1 and G2, which indicated that the CV777 vaccine could cross-protect against G2 epidemic strains, at least to some extent. However, the antigenic and serologic neutralization responses against the $\mathrm{S}$ protein also reflected antigenic differences of twofold between the two PEDV subtypes, based on anti-S PAbs titers. Therefore, future development of vaccines against emerging PEDV strains should involve multiple $S$ proteins containing neutralizing epitopes from both the G1 and G2 subtypes.

Acknowledgments This work was supported by grants from the National Natural Science Foundation of China (grant number 31172350), Innovation Capability Project for Research Institutions of Heilongjiang Province (grant number YC2015D011).

\section{References}

1. Debouck P, Pensaert M (1980) Experimental infection of pigs with a new porcine enteric coronavirus, CV777. J Am Vet Med Assoc 4:219-223

2. Ducatelle R, Coussement W, Charlier G, Debouck P, Hoorens J (1981) Three-dimensional sequential study of the intestinal surface in experimental porcine CV777 coronavirus enteritis. Zentralbl Veterinarmed B 28:483-493

3. Oldham J (1972) Letter to the editor. Pig Farming suppl:72-73

4. Takahashi K, Okada K, Ohshima K (1983) An outbreak of swine diarrhea of a new-type associated with corona-virus like particles in Japan. Nihon Juigaku Zasshi 45:829-832

5. Cima G (2013) Fighting a deadly pig disease. Industry, veterinarians trying to contain PED virus, new to the US. J Am Vet Med Assoc 243(4):469-470

6. Stevenson GW, Hoang H, Schwartz KJ, Burrough EB, Sun D, Madson D, Cooper VL, Pillatzki A, Gauger P, Schmitt BJ et al (2013) Emergence of porcine epidemic diarrhea virus in the United States: Clinical signs, lesions, and viral genomic sequences. J Vet Diagn Invest 25(5):649-654

7. Sun RQ, Cai RJ, Chen YQ, Liang PS, Chen DK, Song CX (2012) Outbreak of porcine epidemic diarrhea in suckling piglets, China. Emerg Infect Dis 18(1):161-163

8. Li W, Li H, Liu Y, Pan Y, Deng F, Song Y, Tang X, He Q (2012) New variants of porcine epidemic diarrhea virus, China. Emerg Infect Dis 18(8):1350-1353

9. Wang XM, Niu BB, Yan H, Gao DS, Yang X, Chen L, Chang HT, Zhao J, Wang CQ (2013) Genetic properties of endemic Chinese porcine epidemic diarrhea virus strains isolated since 2010. Arch Virol 158:2487-2494

10. Park JE, Shin HJ (2014) Porcine epidemic diarrhea virus infects and replicates in porcine alveolar macrophages. Virus Res 191:143-152

11. Pensaert MB, de Bouck P (1978) A new coronavirus-like particle associated with diarrhea in swine. Arch Virol 58(3):243-247

12. Lee DK, Park CK, Kim SH, Lee C (2010) Heterogeneity in spike protein genes of porcine epidemic diarrhea viruses isolated in Korea. Virus Res 149(2):175-182

13. Sun D, Feng L, Shi H, Chen J, Cui X, Chen H, Liu S, Tong Y, Wang Y, Tong G (2008) Identification of two novel B cell epitopes on porcine epidemic diarrhea virus spike protein. Vet Microbiol 131(1-2):73-81
14. Chang SH, Bae JL, Kang TJ, Kim J, Chung GH, Lim CW, Laude H, Yang MS, Jang YS (2002) Identification of the epitope region capable of inducing neutralizing antibodies against the porcine epidemic diarrhea virus. Mol Cells 14(2):295-299

15. Park SJ, Moon HJ, Yang JS, Lee CS, Song DS, Kang BK, Park BK (2007) Sequence analysis of the partial spike glycoprotein gene of porcine epidemic diarrhea viruses isolated in Korea. Virus Genes 35:321-332

16. Bosch BJ, Van Der Zee R, De Haan CA, Rottier PJ (2003) The coronavirus spike protein is a class I virus fusion protein: structural and functional characterization of the fusion core complex. J Virol 77(16):8801-8811

17. Li W, Li H, Liu Y, Pan Y, Deng F, Song Y, Tang X, He Q (2012) New variants of porcine epidemic diarrhea virus, China, 2011. Emerg Infect Dis 18:1350-1353

18. Cheun-Arom T, Temeeyasen G, Srijangwad A, Tripipat $T$, Sangmalee S, Vui DT, Chuanasa T, Tantituvanont A, Nilubol D (2015) Complete genome sequences of two genetically distinct variants of porcine epidemic diarrhea virus in the eastern region of Thailand. Genome Announc 3(3):e00634-e00715. doi:10. 1128/genomeA.00634-15

19. Chen J, Liu X, Shi D, Shi H, Zhang X, Li C, Chi Y, Feng L (2013) Detection and molecular diversity of spike gene of porcine epidemic diarrhea virus in China. Viruses 5:2601-2613

20. Wang J, Zhao P, Guo L, Liu Y, Du Y, Ren S, Li J, Zhang Y, Fan $Y$, Huang B et al (2013) Porcine epidemic diarrhea virus variants with high pathogenicity, China. Emerg Infect Dis 19:2048-2049

21. Sun RQ, Cai RJ, Chen YQ, Liang PS, Chen DK, Song CX (2013) Outbreak of porcine epidemic diarrhea in suckling piglets, China. Emerg Infect Dis 18:161-163

22. Zylicz-Stachula A, Zolnierkiewicz O, Sliwinska K, JezewskaFrackowiak J, Skowron PM (2014) Modified 'one amino acid-one codon' engineering of high GC content TaqII-coding gene from thermophilic Thermus aquaticus results in radical expression increase. Microbial Cell Fact 13:7

23. Fei D, Zhang H, Diao Q, Jiang L, Wang Q, Zhong Y, Fan Z, Ma M (2015) Codon optimization, expression in escherichia coli, and immunogenicity of recombinant chinese sacbrood virus (CSBV) structural proteins VP1, VP2, and VP3. PLoS One 10(6):e0128486. doi:10.1371/journal.pone.0128486

24. Reed LJ, Muench H (1938) A simple method of estimating fifty percent endpoints. Am J Epidemiol 27(3):493-497

25. Song D, Huang D, Peng Q, Huang T, Chen Y, Zhang T, Nie X, He H, Wang P, Liu Q, Tang Y (2015) Molecular characterization and phylogenetic analysis of porcine epidemic diarrhea viruses associated with outbreaks of severe diarrhea in piglets in Jiangxi, China 2013. PLoS One 10(3):e0120310. doi:10.1371/journal. pone. 0120310

26. Jung K, Saif LJ (2015) Porcine epidemic diarrhea virus infection: Etiology, epidemiology, pathogenesis and immunoprophylaxis. Vet J 204:134-143

27. Lee DK, Cha SY, Lee C (2011) The N-terminal region of the porcine epidemic diarrhea virus spike protein is important for the receptor binding. Korean J Microbiol Biotechnol 39:140-145

28. Sun DB, Feng L, Shi HY, Chen JF, Liu SW, Chen HY, Wang YF (2007) Spike protein region (aa 636789) of porcine epidemic diarrhea virus is essential for induction of neutralizing antibodies. Acta Virol 51:149-156

29. Oh J, Lee KW, Choi HW, Lee C (2014) Immunogenicity and protective efficacy of recombinant S1 domain of the porcine epidemic diarrhea virus spike protein. Arch Virol 159:2977-2987

30. Coleman CM, Liu YV, Mu H, Taylor JK, Massare M, Flyer DC, Glenn GM, Smith GE, Frieman MB (2014) Purified coronavirus Spike protein nanoparticles induce coronavirus neutralizing antibodies in mice. Vaccine 32(26):3169-3174 
31. Escriou N, Callendret B, Lorin V, Combredet C, Marianneau P, Février M, Tangy F (2014) Protection from SARS coronavirus conferred by live measles vaccine expressing the spike glycoprotein. Virology 452-453:32-41

32. Chang SH, Bae JL, Kang TJ, Kim J, Chung GH, Lim CW, Laude H, Yang MS, Jang YS (2002) Identification of the epitope region capable of inducing neutralizing antibodies against the porcine epidemic diarrhea virus. Mol Cells 14:295-299

33. Aricescu AR, Owens RJ (2013) Expression of recombinant glycoproteins in mammalian cells: towards an integrative approach to structural biology. Curr Opin Struct Biol 23(3):345-356

34. Nettleship JE, Assenberg R, Diprose JM, Rahman-Huq N, Owens RJ (2010) Recent advances in the production of proteins in insect and mammalian cells for structural biology. J Struct Biol 172:55-65

35. Durocher Y, Perret S, Kamen A (2002) High-level and highthroughput recombinant protein production by transient transfection of suspension-growing human 293-EBNA1 cells. Nucleic Acids Res 30(2):E9

36. Lin CM, Gao X, Oka T, Vlasova AN, Esseili MA, Wang Q, Saif LJ (2015) Antigenic relationships among porcine epidemic diarrhea virus and transmissible gastroenteritis virus strains. J Virol 89(6):3332-3342

37. Wicht O, Li W, Willems L, Meuleman TJ, Wubbolts RW, van Kuppeveld FJ, Rottier PJ, Bosch BJ (2014) Proteolytic activation of the porcine epidemic diarrhea coronavirus spike fusion protein by trypsin in cell culture. J Virol 88:7952-7961

38. Li W, Wicht O, van Kuppeveld FJ, He Q, Rottier PJ, Bosch BJ (2015) A single point mutation creating a furin cleavage site in the spike protein renders porcine epidemic diarrhea coronavirus trypsin-independent for cell entry and fusion. $\mathrm{J}$ Virol 89(15):8077-8081 